\title{
Offener Brief an Herrn Urs P. Gasche
}

\author{
H. Niederer
}

Mit Interesse und einigem Erstaunen habe ich Ihren Artikel (Weltwoche 42, S. 8, 17.10.2002) über die Lösung der Probleme im Gesundheitswesen zur Kenntnis genommen und möchte dazu als Betroffener mit 20 Jahren Berufserfahrung Stellung nehmen. Vorausschicken möchte ich, dass Ihre Einleitung, dass wir viel - je nach Auffassung auch zu viel - für unsere Gesundheit bezahlen, der Realität entspricht. Die Schlüsse, welche Sie allerdings ziehen, entbehren in mehreren Beziehungen eines Realitätsbezugs.

Sie möchten an den Spitälern Pauschalentschädigungen einführen. Es hat in der Schweiz schon Modellversuche gegeben. Diese zeigten, dass wegen des vermehrten administrativen Aufwandes mögliche Kosteneinsparungen grössere Gesamtkosten resultieren. Ob diese angeblich in den USA ausgereiften Kostenberechnungen wirklich effizienter sind, wage ich zu bezweifeln; in den USA haben etwa 15\% der Bevölkerung, darunter wohl viele der sozial Schwächsten, gar keine Krankenkasse. Insgesamt ist das amerikanische Gesundheitswesen genau wegen dieser unvollständigen Versicherung, trotz der höchsten Kosten weltweit, mit Sicherheit nicht das beste. Zudem besteht die Gefahr, dass bei Auftreten von Komplikationen im Falle von Kostenpauschalen Verantwortungen abgeschoben werden: kleinere Spitäler versuchen schwierigere Problemstellungen an grössere Kliniken zu delegieren.

Was Sie über Statistiken zur Erfassung von Komplikationen und Spitalinfektionen behaupten, kann bei sehr vereinfachten Fragestellungen stimmen. Sie können aber erstens keine ungleichen Kliniken miteinander vergleichen (Klinik A führt nur kleine Eingriffe durch, Klinik B, z.B. ein grosses Kantonsspital, hat Kapazitäten auch für aufwendigere Operationen mit den entsprechenden Spezialisten und Labors). Zweitens habe ich die Zahlen einer HMO-Gruppenärztepraxis, in welcher ich früher mitarbeitete, zur Erfassung der Verordnungen der Ärztinnen und Ärzte gesehen: mit diesen Zahlen können keinerlei Aussagen zur Effizienz der einzelnen Ärzte gemacht werden. Als Beispiel möge die Fragestellung gelten, ob ein Arzt, welcher häufiger als ein anderer bestimmte Medikamente zur Verkürzung der Arbeitsunfähigkeit bei Atemwegsinfekten einsetzt, ein schlechterer Arzt ist oder nicht. Der eigentliche Vorteil dieser Medika- mente, nämlich die kürzere Arbeitsunfähigkeit, und damit der direkte volkswirtschaftliche Nutzen wird mit einer solchen Statistik genau nicht erfasst.

Ihre Idee, die Spitäler nicht mehr durch die öffentliche Hand zu finanzieren, würde zu chaotischen Zuständen führen. Private Kliniken gibt es bereits in der Schweiz, diese können aus Rentabilitätsgründen keine allgemeine Notfallversorgung anbieten. Es würden sich auch kaum kostengünstigere Managed-Care-Modelle durchsetzen, diese haben sich, von Ausnahmen abgesehen (meistens in den städtischen Gebieten), nämlich wegen einer schlechteren Effizienz gegenüber vielen Arztpraxen nicht bewährt. Wenn Sie glauben, die Preise für bestimmte medizinische Leistungen willkürlich festlegen zu können, sehen Sie sich doch die Verhältnisse in vielen ländlichen Gebieten unseres Staates an: es herrscht ein unübersehbarer Mangel an Allgemeinärzten. Deren Tarife liegen leider nicht mehr in einem Bereich, welcher das Betreiben einer modernen Praxis erlaubt - viele jungen Ärzte lehnen die Übernahme von Verantwortung bei schlechter Entlöhnung zu Recht ab. Zudem zeigt sich, dass die Qualität der in HMOs angebotenen Leistungen sich oft nicht mit derjenigen in privaten Praxen vergleichen lässt: wenn die Löhne reduziert werden, sinkt damit auch das Engagement unter den angestellten Ärzten.

Würden aber, wie Sie dies im weiteren fordern, die Ärzte in allen Praxen und Kliniken eine Pauschale erhalten, wäre die zwangsläufige Folge, dass sog. schlechte Risiken wenn immer möglich abgeschoben werden. Niemand behandelt alte, gebrechliche oder multifaktoriell kranke Menschen in aufwendiger Art zum gleichen Tarif wie junge, unkomplizierte. Dass die Ärzte interessiert seien, die Patienten nur im Falle von Kostenpauschalen möglichst effizient $\mathrm{zu}$ behandeln, stimmt bei mindestens 90\% der Ärzte nicht die anderen $10 \%$ betreffen die sog. schwarzen Schafe. Wenn Sie glauben, die komplexen Probleme der meisten Patienten mit einfachen Pauschalen zu entschädigen, täuschen Sie sich: die Erfassung und Kategorisierung der Probleme bringt zusätzlichen, kostenintensiven Aufwand. Die Kostensteigerung im Gesundheitswesen ist nicht zuletzt durch massiv angestiegene administrative Kosten erklärt. So sind diese Kosten in den 80er und 90er Jahren ungefähr dreimal 
schneller angestiegen als die effektiven medizinischen Kosten. Dazu kommt, dass diejenigen Patienten, welche bestimmte Leistungen nicht erhalten, zu anderen Ärzten gehen müssen oder wollen, welche die ganze Fragestellung erneut aufwickeln. Dadurch entstehen weitere Kosten sozial Schwache, darunter z.B. viele Ausländer, werden durch solche Massnahmen nicht mehr optimal versorgt, und es könnten viel höhere Kosten bei stationären Behandlungen anfallen -, unter dem Strich also keine Einsparmöglichkeiten.

Wie wollen Sie verhindern, dass Ärzte, welche nach Pauschalen arbeiten (wie z. B. Ärzte in Kliniken oder HMO- Praxen), Arbeit nicht an andere abschieben? Dies kann man entweder durch Kündigung erreichen, oder indem man in Ihrem Modell die Praxis nur noch zu bestimmten Zeiten geöffnet hat. In Deutschland gilt seit 1994 eine Berechnung nach Fallkostenpauschalen für Arztpraxen. Das Resultat zeigt sich in einer längeren Feriendauer der Ärzte. Kein Arzt bietet mehr Sprechstunden an, wenn er für seine Arbeit wegen der erreichten Budget-Limite keine Rechnung stellen kann. Die Folge davon sind längere Arbeitszeiten an öffentlichen Spitälern, welche kaum effizienter arbeiten als private Arztpraxen. Insgesamt sind die Kosten im Gesundheitswesen in Deutschland seit Einführung dieses Modells laut Angaben dort tätiger Ärzte gestiegen.

Die sog. Vertragsfreiheit wird oft diskutiert. Wenn Sie diese einführen wollen, werden Sie ein Modell erleben, wie wir es in der Zahnarztmedizin kennen. Sie können dazu den Artikel von Herrn M. Schneider in der Weltwoche Nr. 23 2002, S. 32 ff. lesen: die Zahnärzte haben die Tarife durch eine Zunftpolitik hochgehalten, es werden nicht mehr Berufsleute ausgebildet als zwingend erforderlich, das Angebot deckt knapp die Nachfrage. Wollen Sie solche Zustände in unserem Gesundheitswesen? Wie wollen Sie unter solchen Umständen den Leuten erklären, warum ein Obligatorium für eine Krankenversicherung besteht? Genauso gefährlich ist auch die Vorstellung der SVP, mehrere Leistungen aus der Grundversicherung herauszunehmen. Zweifels- ohne klammert die Vertragsfreiheit die Arztwahl nicht aus, wohl aber die administrativen Kosten - diese werden wohl kaum freiwillig von den Ärzten oder den Patienten übernommen.

Was die Arztwahl bei Operationen angeht, haben Sie nur den einen Teil der Probleme der Chirurgie angesprochen. Wenn das neue Tarifmodell verwirklicht wird, werden viele Eingriffe nicht mehr kostendeckend entschädigt. Wenn ein Patient z. B. für eine Venen-Operation gar keinen Arzt mehr findet (solche Verhältnisse gibt es z.T. bereits in Deutschland), kann von besserer Arztwahl keine Rede sein. Noch verheerendere Verhältnisse erwarten einen Patienten mit einer Blinddarmentzündung.

Ich bezweifle, ob Ihre Vorschläge, das sog. Massnahmenpaket überhaupt realisierbar ist und nicht zu noch viel höheren Kosten führen würde. Wenn Sie Ärztinnen und Ärzte mit Pauschalen für erbrachte Leistungen entschädigen wollen, sind viel mehr Berufsleute erforderlich (die von einer HMO-Gruppenärztepraxis erbrachten Leistungen könnten in einer privaten Gruppenärztepraxis mit weniger Personal, bei allerdings höherer Entschädigung der einzelnen Mitarbeiter ebenfalls erbracht werden). Gestatten Sie mir eine Bemerkung: ich möchte nicht in einem System mitarbeiten, in welchem man unnötige Leistungen anbietet, weil man per Vertrag dazu gezwungen ist - ich möchte aber genausowenig wegen eines solchen Vertrages eine zu grosse Zahl von Patienten versorgen müssen und dann eine qualitativ minderwertige Medizin anbieten.

Ein ganz wesentlicher Aspekt ist an dieser Stelle noch nicht diskutiert worden: wenn ärztliche Leistungen wegen der fehlenden Kostengutsprache von Kassen nicht mehr erbracht werden, könnten auch unabschätzbare Kostenaufwendungen durch Gerichtsverfahren anfallen.

Grundsätzlich sind Vorschläge von Journalisten zu begrüssen; es wäre allerdings begrüssenswert, wenn sich die Medienfachleute ein bisschen über die reell existierenden Verhältnisse informieren würden - dies habe ich in Ihren Ausführungen leider vermisst. 